\title{
Hepatitis B and Renal Disease
}

\author{
Tak Mao Chan
}

Published online: 14 April 2010

(C) The Author(s) 2010. This article is published with open access at Springerlink.com

\begin{abstract}
Glomerulonephritis is an important extrahepatic manifestation of chronic hepatitis B virus (HBV) infection. The uncommon occurrence, variability in renal histopathology, and heterogeneity in clinical course present challenges in clinical studies and have resulted in a relative paucity of data and uncertainty with regard to the optimal management of HBV-related glomerular diseases. The advent of nucleos $(\mathrm{t})$ ide analogue medications that effectively suppress HBV replication has markedly altered the clinical outcomes of kidney transplant recipients with HBV infection, but the emergence of drug resistance is an escalating problem. This article reviews the recent knowledge of the pathogenesis and treatment of HBV-related membranous nephropathy, and discusses the management of hepatitis B in kidney transplant recipients, which is continuously evolving.
\end{abstract}

Keywords Hepatitis B · Kidney transplantation

\section{Introduction}

The hepatitis B virus (HBV) has a complex relationship to kidney diseases. Chronic HBV infection is an etiologic factor in secondary glomerular diseases. From an opposite perspective, the HBV carrier status has a critical impact on the clinical management of kidney transplant recipients and patients with renal diseases who are treated with immunosuppressive drugs.

T. M. Chan $(\bowtie)$

Department of Medicine, University of Hong Kong,

Queen Mary Hospital,

Pokfulam,

Hong Kong, China

e-mail: dtmchan@hku.hk
HBV is not directly cytopathic to hepatocytes. The host immune response, especially via virus-specific cytotoxic $\mathrm{T}$ lymphocytes, is the basis for hepatocellular damage as well as viral clearance. Neonatal exposure to HBV when the immune system is immature results in minimal acute hepatitis, but this is followed by chronic infection in $90 \%$ of subjects, and contributes to the bulk of chronic HBV carriers. After entering hepatocytes by endocytosis, the partially double-stranded viral genomic DNA is transported into the nucleus, where it is converted to covalently closed circular DNA, which serves as a template for transcription of viral mRNAs, which in turn are used for viral replication through reverse transcription and the production of viral DNA polymerase and other viral proteins. Intrahepatic covalently closed circular DNA thus accounts for the persistence of infection. About 350 million people worldwide have chronic HBV infection. The importance of HBV in clinical nephrology increases markedly in areas with endemic infection such as South East Asia, where the proportion of chronic HBV carriers can exceed $10 \%$ in the general population. In this regard, data from a recent series that included 390 patients with membranous nephropathy showed that HBV was the underlying cause in $12 \%$ of patients [1]. This article reviews the clinical and therapeutic aspects of HBV-related glomerulonephritis focusing on membranous nephropathy, kidney transplantation with HBsAg-positive donors, and the evolving management of hepatitis B in kidney transplant recipients.

\section{HBV-related Glomerulonephritis}

Extrahepatic manifestations of HBV infection can present as glomerulonephritis, vasculitis, or reactive arthritis. Glomerular disease is more common in children than 
adults, and in men than women [2]. The more common renal histopathologies include membranous nephropathy and membranoproliferative glomerulonephritis. An association with IgA nephropathy has been reported, and recent reports also suggest a possible link with focal segmental glomerulosclerosis [3].

\section{Pathogenic Mechanisms}

Both viral and host factors are involved in pathogenesis. An association with HLA genes has been reported, indicating the impact of genetic predisposition [4]. The main pathogenic mechanism in HBV-related glomerular diseases is through the deposition of immune complexes in the glomerulus. The immune complexes are comprised of viral antigens and the antibodies that these antigens invoke from the host. Whether these immune complexes are formed in situ or are derived from circulating immune complexes being trapped in the glomerulus remains controversial. Various HBV antigens including hepatitis B surface antigen (HBsAg), hepatitis B early antigen ( $\mathrm{HBeAg}$ ), and hepatitis $B$ core antigen ( $\mathrm{HBcAg}$ ) have been demonstrated in the glomeruli of patients with HBV-related glomerulonephritis, and the detection of covalently closed circular DNA in renal tissue has also been reported [5]. Immune deposition occurs predominantly in the subepithelial region but can also involve the mesangial and occasionally subendothelial areas, depending on the size of the antigens and immune complexes. It has been speculated that the low molecular weight of $\mathrm{HBeAg}\left(3 \times 10^{5} \mathrm{Da}\right)$ might account for its ability to traverse the glomerular basement membrane and thus the formation of subepithelial immune deposits [6]. The observed association between remission of proteinuria and clearance of $\mathrm{HBeAg}$ also provides indirect evidence that the antigen is involved in pathogenesis. The immune complexes then activate complements and glomerular injury occurs via the formation of membrane attack complex and other downstream events such as the induction of proteases, oxidation injury, and disruption of cytoskeleton [7, 8]. Investigations on renal gene expression profile with microarrays, in transgenic mice that expressed HBsAg and $\mathrm{HBcAg}$ in the cytoplasm of renal tubular epithelial cells but without replication of the whole virus, have revealed upregulation of complement and coagulation pathways and acute phase response genes, and reduced circulating $\mathrm{C} 3$ levels [9].

It has been reported that the sera of patients with chronic HBV infection could induce apoptosis in cultured HK-2 cells, a cell line for the study of human proximal renal tubular epithelial cells, via up-regulation of Fas gene expression [10]. Furthermore, the induction of apoptosis correlated with the level of circulating HBV DNA, and HBV carriers also showed a higher circulating level of transforming growth factor-beta, a growth factor implicated in the potentiation of apoptosis and renal fibrosis. These preliminary data suggest the presence of serum factor(s) in HBV carriers which could alter renal tubular cell function, and should be confirmed using samples from patients with documented nephritis and in podocytes.

HBV can be classified into eight major genotypes based on genome sequence divergence. The impact of viral genetics was recently investigated in a study that included two pediatric and four adult Japanese patients, five of whom had membranous nephropathy, and one with membranoproliferative glomerulonephritis [11]. All the patients were HBeAg-positive and had high circulating HBV DNA levels. The investigators did not find any association between nephropathy and mutations in the HBV genome. However, the genotyping results were interesting. Previous studies showed that genotype $\mathrm{C}$ was predominant among local subjects with chronic hepatitis B, whereas genotype A accounted for only $1.7 \%$ [12]. Yet complete viral genome sequencing showed that among the patients with nephropathy four had HBV genotype A1/A2, whereas two were infected with genotype $\mathrm{C} 2$. A high prevalence of genotype A in patients with HBV-related nephropathy had been reported by other investigators [13, 14]. Whether HBV genotype A may indeed be more likely to lead to renal manifestations compared with other genotypes, and the mechanisms accounting for such difference, require further investigations.

Clinicopathologic Features of HBV-related Membranous Nephropathy and Membranoproliferative

Glomerulonephritis

Patients with HBV-related membranous nephropathy typically present with proteinuria, which could be in the nephrotic range, and microscopic hematuria. Impaired renal function is more common in patients with membranoproliferative glomerulonephritis. The natural history of HBV-related membranous nephropathy appears different between children and adults. In contrast to pediatric subjects, in whom spontaneous remission of proteinuria is common and the renal function is often well preserved, adult patients are more likely to have progressive disease and up to one third of patients might eventually develop renal failure $[13,15,16]$. A temporal relationship between increased hepatitic activity and deterioration of proteinuria has been observed in some patients [17], and could be associated with cryoglobulinemia.

HBV-related membranous nephropathy is characterized by thickened capillary wall and glomerular basement membrane on light microscopy. Although this feature could be subtle in the early stage, the capillary wall can assume a rigid appearance in advanced disease. Immunofluorescent staining and electron microscopy demonstrate granular IgG, 
$\mathrm{C} 3$, and some IgM staining in the subepithelial region along the glomerular basement membrane accompanied by extensive effacement of the podocyte foot processes, and in some cases viral particles in various locations within the glomerulus. Mesangial abnormalities are more common in secondary membranous nephropathy compared with the idiopathic form. Mesangial expansion and capillary wall thickening resulting in a lobular appearance of the glomerular tuft characterize the light microscopic findings in membranoproliferative glomerulonephritis. The capillary wall also demonstrates a double-contour appearance and hypercellularity with interpositioning of cells. The latter may include infiltrating monocytes and neutrophils. Immune deposits containing IgG, complement components, and IgM appear granular on immunofluorescent staining, and are located in the subendothelial, mesangial, and paramesangial areas. In addition to these electron-dense deposits, which could also be present in the subepithelial region, albeit in smaller amounts, electron microscopy also shows subendothelial expansion and the formation of new basement membrane material, which accounts for the double-contour appearance on light microscopy. The subendothelial and mesangial immune deposits trigger complement activation and increased local expression of inflammatory and chemotactic mediators, leading to the infiltration of inflammatory cells.

\section{Treatment of HBV-related Membranous Nephropathy}

Much of the data on the treatment of HBV-related glomerular diseases came from patients with membranous nephropathy, the most common histologic presentation, whereas the data on membranoproliferative glomerulonephritis or focal segmental glomerulosclerosis remain largely anecdotal. Treatment with interferon [14, 18-25] or lamivudine [17, 26-28] has been reported to lead to a reduction of proteinuria in patients, mostly children, with HBV-related membranous nephropathy.

Conventional and pegylated interferon- $\alpha$ possess both immunoregulatory and antiviral effects [29]. Interferon- $\alpha$ activates cellular pathways that lead to breakdown of viral RNA and enhances cell-mediated immune response toward hepatocytes infected with HBV. Interferon treatment given for 4-12 months was associated with sustained remission of proteinuria in $20 \%$ to $100 \%$ of patients, clearance of $\mathrm{HBeAg}$ in $20 \%$ to $80 \%$, and a drop-out rate of $10 \%$ to $15 \%$. Resolution of proteinuria was often associated with clearance of $\mathrm{HBeAg}$ and/or HBsAg, and usually occurred within 6 months of seroconversion [13, 23, 24]. However, most of the reported data were anecdotal reports or data from small series. Bias from selective publication of positive results remains possible. There is also a general impression that the results in adults may be less favorable compared with children.
Nucleos(t)ide analogues such as lamivudine, telbivudine, adefovir, entecavir, or tenofovir suppress HBV replication through their inhibitory effect on viral DNA polymerase. Because the deposition of immune complexes within the glomerulus is perceived to play a pivotal role in the pathogenesis of HBV-related nephropathy, reducing the quantity of viral antigens and thereby reducing immune complex deposition in the kidneys should (in theory) ameliorate kidney damage. Compared with interferon, nucleos $(\mathrm{t})$ ide analogues offer the advantages of convenient administration and high tolerability, but often require longterm administration and could result in the selection of drug-resistant HBV strains. Adefovir should be used with caution in patients with renal impairment in view of its nephrotoxicity, which is mediated through inhibitioin of mitochondrial DNA replication resulting in disruption of normal mitochondrial respiratory function in proximal renal tubular epithelial cells. There are also uncommon reports of renal tubular toxicity with tenofovir [30]. Even fewer data exist regarding nucleos(t)ide analogues than interferon on HBV-related nephropathy. The factors predictive of treatment efficacy and the selection criteria for treatment also remain undefined. Data from a recent report on 10 patients with HBV-related membranous nephropathy showed that lamivudine treatment was associated with complete resolution of proteinuria in six patients [28]. However, the concomitant use of angiotensin-converting enzyme inhibitors, angiotensin II receptor blockers, and antihypertensive medications precluded definitive conclusions on the renal effect of antiviral treatment. Future studies on the effect of antiviral therapy on proteinuria or renal survival should thus include concurrent controls with comparable exposure to these potential confounders.

The renal prognosis is distinctly different between pediatric and adult patients, with the incidence of chronic kidney disease reported as less than $3 \%$ in children and up to $30 \%$ in adult patients $[13,15]$. The overall data suggest that a favorable renal response to antiviral treatment seems more likely in pediatric patients than adults, and in patients when renal manifestations are accompanied by increased viral replication, a high HBV DNA level, and hepatitic flare.

\section{HBV and Kidney Transplantation}

The prevalence of HBsAg seropositivity in the general population varies from less than $1 \%$ in nonendemic areas to more than $10 \%$ in regions with endemic infection. Similarly, the prevalence of HBV carriers among patients on dialysis varies from about $1 \%$ in the United States to more than $10 \%$ in some Asian countries [31,32]. The use of kidneys from HBsAg-positive donors demands caution to avoid transmission of infection via the allograft. HBsAg- 
positive kidney transplant recipients are at risk of enhanced viral replication and exacerbation of the liver disease consequent to immunosuppression.

\section{HBsAg-positive Kidney Donors}

The use of kidneys from HBsAg-positive donors has been a controversial issue. In regions with endemic HBV infection, HBsAg-positive subjects account for a significant donor pool. With the availability of effective antiviral nucleos(t)ide analogues, little disagreement exists on kidney transplantation from an HBsAg-positive donor to an HBsAg-positive recipient. However, there has been concern over the safety of transplanting from an HBsAgpositive donor to a recipient who is anti-HBs-positive, for fear that the preexisting immunity might not be adequate to combat the virus load transferred with the transplanted kidney in the face of heavy immunosuppression. Data from relatively small series, from our unit and others, suggested that it was safe to transplant kidneys from HBsAg-positive donors to anti-HBs-positive recipients [33]. A recent report examined the outcomes of 65 antiHBs-positive subjects who had received kidney transplants from HBsAg-positive deceased donors, and compared them with 308 anti-HBs-positive subjects who had received kidney transplants from HBsAg-negative deceased donors, with an average follow-up of more than 3 years [34•]. All kidney transplant recipients were given 400 units of antiHBV immunoglobulin at the time of surgery, and those who had received HBsAg-positive kidneys had a second dose 1 month later. When the donor was HBV DNA-positive by quantitative polymerase chain reaction assay, the recipients were given a prolonged course of anti-HBV immunoglobulin at 400 units weekly for 3 months and lamivudine for 6 months. Seroconversion to HBsAg-positivity occurred in two patients, one in the donor $\mathrm{HBsAg}$-positive group (the donor was HBV DNA-negative) at 3 months and the other in the donor HBsAg-negative group (the donor was also anti-HBc-negative) within the first month. Baseline antiHBs levels in the two kidney recipients were $63.6 \mathrm{IU} / \mathrm{L}$ and 4.6 IU/L, respectively. The second patient seroconverted back to become HBsAg-negative and anti-HBs-positive at 6 months after transplantation. Different investigators have reported occult HBV infection in patients on long-term dialysis, which can occur even in the presence of anti-HBs $[35,36]$. In this study, potential kidney recipients were apparently monitored serially for their HBV DNA status, although the sensitivity of detection was not stated. The absence of viral gene sequence data precluded definitive conclusions on whether the HBsAg seroconversions were caused by de novo HBV infection. Nevertheless, the data reaffirm the overall safety of transplanting kidneys from HBsAg-positive donors to anti-HBs-positive recipients, irrespective of whether the immunity has resulted from prior natural infection or vaccination. However, the role of perioperative anti-HBV immunoglobulin and antiviral medications remains unclear.

\section{HBV Infection in Kidney Transplant Recipients}

HBV-related liver complications can present as de novo infection, acute flare in patients with chronic infection, fibrosing cholestatic hepatitis, chronic hepatitis, cirrhosis, or hepatocellular carcinoma. The adverse impact of HBV infection on clinical outcomes has been reported by different investigators [37-40]. Surveillance for hepatocellular carcinoma with regular $\alpha$-fetoprotein measurement and liver ultrasonogram is essential in view of the much improved prognosis conferred by the diagnosis of early tumor compared with advanced disease. Prevention and surveillance of liver complications in HBsAg-positive kidney transplant recipients is a lifelong process that starts before kidney transplantation (Table 1).

Data from patients with hepatitis $\mathrm{C}$ infection showed that interferon treatment could lead to a high incidence of renal allograft failure [41]. Thus, prior to the advent of nucleos $(t)$ ide analogues, the lack of effective and safe treatment had added to the clinical challenge in the management of hepatitis B in kidney transplant recipients. The incidence of chronic liver disease in HBsAg-positive kidney transplant recipients was up to $80 \%$, and about $60 \%$ of the mortalities were from liver complications [37-40]. Our own data showed that in the prelamivudine era, HBsAg-positive kidney transplant recipients had excessive early mortality rates within the first 2 years after transplantation compared with HBsAg-negative controls, and the deaths were from severe hepatitic flares brought about by heavy immunosuppression [40]. Although $43 \%$ of the HBsAg-positive patients were $\mathrm{HBeAg}$-positive at the time of kidney transplantation, $\mathrm{HBeAg}$ status by itself could not serve as a reliable predictor of liver complications, and severe complications could also occur in HBeAg-negative subjects.

\section{Monitoring and Treatment}

The management of HBsAg-positive kidney transplant recipients entails monitoring for the early diagnosis of liver complications, especially with regard to hepatitic flare and hepatocellular carcinoma, and the prevention of cirrhosis. Hepatitic flares are preceded by accelerated viral replication and increasing circulating HBV DNA levels, and the risk of flare varies according to the level of concurrent immunosuppression. Fibrosing cholestatic hepatitis, an uncommon but serious complication occurring in heavily immunosuppressed patients, is characterized by relatively mild elevations in serum transaminase levels but markedly elevated 
Table 1 Preventive and surveillance measures against HBV related complications in kidney transplant recipients

\begin{tabular}{ll}
\hline Phase & Preventive/surveillance measures \\
\hline $\begin{array}{l}\text { Before kidney } \\
\text { transplantation }\end{array}$ & 1. Hepatitis B vaccination in HBsAg-negative anti-HBs-negative patients with chronic kidney disease \\
2. Monitor ductal and parenchymal enzymes and $\alpha$-fetoprotein levels, and perform periodic liver imaging, in \\
HBsAg-positive patients \\
3. Assay HBeAg and anti-HBe status, and HBV DNA level, when there is evidence of hepatitis or when \\
considering treatment, and preferably immediately before kidney transplantation \\
4. Liver biopsy for assessment of cirrhosis or hepatitis to be considered judiciously in view of the increased risk \\
of bleeding \\
$\begin{array}{ll}\text { 1. Safe to transplant kidney from HBsAg-positive donor to anti-HBs-positive recipient; role of perioperative anti- } \\
\text { HBV immunoglobulin unclear }\end{array}$ \\
$\begin{array}{l}\text { 2. HBsAg-positive kidney transplant recipients should be prescribed nucleos(t)ide analogue treatment either } \\
\text { a) prophylactically starting at the time of kidney transplantation, or } \\
\text { b) preemptively when the level of HBV DNA increases, with or without abnormal transaminase levels; a } \\
\text { preemptive approach necessitates frequent monitoring of HBV DNA level }\end{array}$ \\
$\begin{array}{l}\text { 1. Monitor ductal and parenchymal enzymes and } \alpha \text {-fetoprotein levels, and perform periodic liver imaging, in } \\
\text { HBster kidney transplantation }\end{array}$ \\
$\begin{array}{l}\text { 2. Measure HBV DNA level with varying frequency according to the degree of immunosuppression } \\
\text { 3. Measure HBV DNA level when there is evidence of hepatitis or when drug resistance is suspected }\end{array}$
\end{tabular}

HBeAg hepatitis B early antigen, HBsAg hepatitis B surface antigen, $H B V$ hepatitis B virus

HBV DNA levels and a rapid progression to liver failure if untreated. Serial quantitation of HBV DNA titer is thus important for the early diagnosis of impending serious acute liver complications. It has been shown that, compared with prophylactic or preemptive treatment, salvage treatment given after the onset of hepatic dysfunction was associated with inferior clinical outcomes [42].

We have reported that serial monitoring of circulating HBV DNA levels combined with nucleos(t)ide analogue treatment in patients who showed an increasing viral load was highly efficacious in reducing liver complications and markedly improved the survival of HBsAg-positive kidney transplant recipients [40]. If frequent monitoring of HBV DNA level is not available, it is also acceptable to start treatment prophylactically at the time of kidney transplantation in view of the frequent occurrence of hepatitic flare within the first 2 years after kidney transplantation in HBsAg-positive subjects. Our latest results show that nucleos(t)ide analogue treatment is associated with improved long-term patient survival in $\mathrm{HBsAg}$-positive kidney transplant recipients, from $40 \%$ to $70 \%$ at 20 years, and that the patient survival rates are similar between HBsAg-positive and HBsAg-negative subjects transplanted in the present era of nucleos(t)ide analogue treatments up to 10 years after transplantation (unpublished data). The beneficial effect of antiviral treatment on patient survival was confirmed in a series of 27 Korean patients who had been treated with lamivudine when their HBV DNA levels increased after kidney transplantation [43]. The 10-year patient survival rates increased from $50 \%$ in those without treatment to $85 \%$ in lamivudine-treated subjects.
In our previous studies, we started nucleos $(\mathrm{t})$ ide analogue treatment when HBV DNA level was at or above $10^{5}$ copies/mL irrespective of normal transaminase levels, or in the range of $10^{2}-10^{5}$ copies $/ \mathrm{mL}$ when the transaminase level was elevated. The HBV DNA threshold for treatment in nonimmunosuppressed subjects is constantly evolving. In patients presenting with severe hepatitic flare, treatment is recommended as long as HBV DNA is detectable. By contrast, the HBV DNA threshold for treatment has been set at $2,000 \mathrm{IU} / \mathrm{mL}$ or about $10^{4}$ copies $/ \mathrm{mL}$ in subjects with compensated cirrhosis and normal transaminase levels [44•]. In patients with precirrhotic liver disease, correlations have been observed between the level of HBV DNA and the risk of cirrhosis, hepatocellular carcinoma, and liverrelated mortaliy, and the risk ratio starts to increase when the HBV DNA level exceeds $10^{4}$ copies $/ \mathrm{mL}$. Poor outcomes are predicted by persistently high HBV DNA levels and the persistence of $\mathrm{HBeAg}[45,46]$. Treatment decisions are thus informed by transaminase levels, HBV DNA levels, $\mathrm{HBeAg}$ status, and liver histology, with HBV DNA levels of $10^{4}-10^{5}$ copies $/ \mathrm{mL}$ regarded by most as the threshold.

\section{Lamivudine Resistance}

Prolonged treatment with nucleos(t)ide analogues can lead to the selection of drug-resistant HBV variants. Avoidance of this problem might be accomplished with discontinuation of treatment after stabilization, but this is feasible in not more than half of the patients [40, 47]. Most patients thus require continued treatment for an indefinite duration. 
We observed a cumulative drug resistance rate of $48 \%$ in kidney transplant recipients who had received lamivudine treatment for an average duration of 69 months [48]. Drug resistance could not be predicted by patient demographics, baseline $\mathrm{HBeAg}$ status or $\mathrm{HBeAg}$ seroconversion, or $\mathrm{HBV}$ genotype, but was associated with a high pretreatment HBV DNA titer, similar to what had been observed in nonimmunosuppressed individuals. Following the emergence of drug resistance, both the HBV DNA and the transaminase levels showed a biphasic longitudinal profile, so that an initial increase was followed by spontaneous gradual improvement, with peak transaminase levels appearing later than those of HBV DNA. About $80 \%$ of patients developed hepatitic flares. It is noteworthy that the peak transaminase level after the emergence of lamivudine resistance was more than twice the level at baseline, and some patients might develop potentially fatal complications during flare. Thus treatment with alternative medications are indicated. Similar to our experience, Korean investigators have reported a 53\% cumulative resistance rate in kidney transplant recipients after 5 years of lamivudine treatment, and in this series two patients died from hepatitic flares after the emergence of drug resistance [43].

We recently reported our preliminary experience in the management of kidney transplant recipients who developed lamivudine resistance [49]. The data showed that, whereas alternative agents such as entecavir or adefovir were mostly effective, the suppression of viral load was less rapid compared with the rate of HBV DNA decline in treatmentnaïve subjects. We used adefovir only in patients with serum creatinine below $150 \mathrm{umol} / \mathrm{L}$, and their kidney function remained stable during short-term follow-up.

Similar to kidney transplant recipients, HBsAg-positive patients with other kidney diseases that require immunosuppressive treatment are exposed to the risk of viral activation and hepatitic flare. We have adopted a similar protocol of HBV DNA monitoring and preemptive treatment in patients with severe lupus nephritis who require combination treatment with high-dose corticosteroid and a cytotoxic agent, and have shown that this can effectively prevent or abort hepatitic flares [50].

\section{Conclusions}

Although the incidence of new infection is decreasing with the increasing practice of hepatitis $B$ vaccination, HBV infection is still a profound global problem and an important cause of secondary glomerular diseases. The data suggest that patients presenting with proteinuria accompanied by active viral replication, a high viral load, and active hepatitis are more likely to show renal benefit following antiviral treatment. More data from adults, and on nucleos(t)ide analogues, are awaited. By contrast, data are accumulating to confirm the efficacy of nucleos(t)ide analogue treatment in improving the survival of HBsAgpositive kidney transplant recipients, given either preemptively when HBV DNA level is increasing or prophylactically from the time of transplantation. Quantitation of circulating HBV DNA level facilitates the early diagnosis of hepatitic flare and the emergence of resistance. The management of drug resistance, and the threshold HBV DNA level for treatment, continue to evolve with increasing clinical experience.

Disclosure No potential conflict of interest relevant to this article was reported.

Open Access This article is distributed under the terms of the Creative Commons Attribution Noncommercial License which permits any noncommercial use, distribution, and reproduction in any medium, provided the original author(s) and source are credited.

\section{References}

Papers of particular interest, published recently, have been highlighted as:

- Of importance

1. Zeng $\mathrm{CH}$, Chen HM, Wang RS, et al.: Etiology and clinical characteristics of membranous nephropathy in Chinese patients. Am J Kidney Dis 2008, 52:691-698.

2. Levy M, Chen N: Worldwide perspective of hepatitis B-associated glomerulonephritis in the 80s. Kidney Int 1991, Suppl 35:S24 S33.

3. Khaira A, Upadhyay BK, Sharma A, et al.: Hepatitis B virus associated focal and segmental glomerular sclerosis: report of two cases and review of literature. Clin Exp Nephrol 2009, 13:373-377.

4. Park MH, Song EY, Ahn C, et al.: Two Subtypes of hepatitis B virus-associated glomerulonephritis are associated with different HLA-DR2 alleles in Koreans. Tissue Antigens 2003, 62:505-511.

5. Chen $\mathrm{L}, \mathrm{Wu} \mathrm{C}$, Fan $\mathrm{X}$, et al.: Replication and infectivity of hepatitis $\mathrm{B}$ virus in HBV-related glomerulonephritis. Int $\mathrm{J}$ Infect Dis 2009, 13:394-398.

6. Takekoshi Y, Tochimaru H, Nagata Y, Itami N: Immunopathogenetic mechanisms of hepatitis B virus-related glomerulopathy. Kidney Int 1991, Suppl 35:S34-S39.

7. Nangaku M, Couser MG: Mechanisms of immune-deposit formation and the mediation of immune renal injury. Clin Exp Nephrol 2005, 9:183-191.

8. Topham PS, Haydar SA, Kuphal R, et al.: Complement-mediated injury reversibly disrupts glomerular epithelial cell actin microfilaments and focal adhesions. Kidney Int 1999, 55:1763-75.

9. Ren J, Wang L, Chen Z, et al.: Gene expression profile of transgenic mouse kidney reveals pathogenesis of hepatitis B virus associated nephropathy. J Med Virol 2006, 78:551-560.

10. Deng CL, Song XW, Liang HJ, et al.: Chronic hepatitis B serum promotes apoptotic damage in human renal tubular cells. World $\mathrm{J}$ Gastroenterol 2006, 12:1752-1756. 
11. Kusakabe A, Tanaka Y, Kurbanov F, et al.: Virological features of hepatitis B virus-associated nephropathy in Japan. J Med Virol 2007, 79:1305-1311.

12. Orito E, Ichida T, Sakugawa H, et al.: Geographic distribution of hepatitis B virus (HBV) genotype in patients with chronic HBV infection in Japan. Hepatology 2001, 34:590-594.

13. Gilbert RD, Wiggelinkhuizen J: The clinical course of hepatitis B virus-associated nephropathy. Pediatr Nephrol 1994, 8:11-14.

14. Bhimma R, Coovadia HM, Adhikari M: Hepatitis B virusassociated nephropathy in black South African children. Pediatr Nephrol 1998, 12:479-484.

15. Lai KN, Li PK, Lui SF, et al.: Membranous nephropathy related to hepatitis B virus in adults. N Engl J Med 1991, 324:1457-1463.

16. Lin CY: Hepatitis B virus-associated membranous nephropathy: clinical features, immunologic profiles and outcomes. Nephron 1990, 55:37-44.

17. Okuse C, Yotsuyanagi H, Yamada N, et al.: Successful treatment of hepatitis B virus-associated membranous nephropathy with lamivudine. Clin Nephrol 2006, 65:53-56.

18. Garcia G, Scullard G, Smith C, et al.: Preliminary observation of hepatitis B-associated membranous glomerulonephritis treated with leukocyte interferon. Hepatology 1985, 5:317-320.

19. Mizushima N, Kanai K, Matsuda $\mathrm{H}$, et al.: Improvement of proteinuria in a case of hepaittis B-associated glomerulonephritis after treatment with interferon. Gastroenterology 1987, 92:524-526.

20. de Man RA, Schalm SW, van der Heijden AJ, et al.: Improvement of hepatitis B-associated glomerulonephritis after antiviral combination therapy. J Hepatol 1989, 8:367-372.

21. Lisker-Melman M, Webb D, Di Bisceglie AM, et al.: Glomerulonephritis caused by chronic hepatitis B virus infection: treatment with recombinant human alpha-interferon. Ann Intern Med 1989, 111:479-483

22. Wong $\mathrm{SN}, \mathrm{Yu} \mathrm{EC}$, Lok AS, et al.: Interferon treatment for hepatitis B-associated membranous glomerulonephritis in two Chinese children. Pediatr Nephrol 1992, 6:417-420.

23. Conjeevaram HS, Hoofnagle JH, Austin HA, et al.: Long-term outcome of hepatitis B virus-related glomerulonephritis after therapy with interferon alfa. Gastroenterology 1995, 109:540-546.

24. Lin CY: Treatment of hepatitis B virus-associated membranous nephropathy with recombinant alpha-interferon. Kidney Int 1995, 47:225-230

25. Chung DR, Yang WS, Kim SB, et al.: Treatment of hepatitis B virus associated glomerulonephritis with recombinant human alpha interferon. Am J Nephrol 1997, 17:112-117.

26. Connor FL, Rosenberg AR, Kennedy SE, Bohane TD: HBV associated nephrotic syndrome: Resolution with oral lamivudine. Arch Dis Child 2003, 88:446-449.

27. Filler G, Feber J, Weiler G, Le Saux N: Another case of HBV associated membranous glomerulonephritis resolving on lamivudine. Arch Dis Child 2003, 88:460.

28. Tang S, Lai FM, Lui YH, et al.: Lamivudine in hepatitis B-associated membranous nephropathy. Kidney Int 2005, 68:1750-1758.

29. Cooksley WG, Piratvisuth T, Lee SD, et al.: Peginterferon alpha-2a $(40 \mathrm{kDa})$ : an advance in the treatment of hepatitis B e antigenpositive chronic hepatitis B. J Viral Hepat 2003, 10:298-305.

30. Karras A, Lafaurie M, Furco A, et al.: Tenofovir-related nephrotoxicity in human immunodeficiency virus-infected patients: three cases of renal failure, Fanconi syndrome, and nephrogenic diabetes insipidus. Clin Infect Dis 2003, 36:1070-1073.

31. Finelli L, Miller JT, Tokars JI, et al.: National surveillance of dialysis-associated diseases in the United States, 2002. Semin Dial 2005, 18:52-61.

32. Johnson DW, Dent H, Yao Q, et al.: Frequencies of hepatitis B and $\mathrm{C}$ infections among haemodialysis and peritoneal dialysis patients in Asia-Pacific countries: analysis of registry data. Nephrol Dial Transplant 2009, 24:1598-1603.
33. Chan MK, Chang WK: Renal transplantation from $\mathrm{HBsAg}$ positive donors to HBsAg negative recipients. Br Med J 1988, 297:522-523.

34. - Jiang $\mathrm{H}, \mathrm{Wu} \mathrm{J}$, Zhang $\mathrm{X}$, et al.: Kidney transplantation from hepatitis B surface antigen positive donors into hepatitis B surface antibody positive recipients: a prospective nonrandomized controlled study from a single center. Am J Transplant 2009, 9:18531858. This is the largest study to date confirming the safety of transplanting kidneys from HBsAg-positive donors to anti-HBspositive recipients. However, since all recipients were given anti$H B V$ immunoglobulin and some were also prescribed lamivudine, the efficacy or necessity of these agents to prevent de novo $H B V$ infection remains unclear.

35. Minuk GY, Sun DF, Greenberg R, et al.: Occult hepatitis B virus infection in a North American adult hemodialysis patient population. Hepatology 2004, 40:1072-1077.

36. Motta JS, Mello FC, Lago BV, et al.: Occult hepatitis B virus infection and lamivudine-resistant mutations in isolates from renal patients undergoing hemodialysis. J Gastroenterol Hepatol 2009, 25:101-106.

37. Rao KV, Kasiske BL, Andersen WR: Variability in the morphological spectrum and clinical outcome of chronic liver disease in hepatitis $\mathrm{B}$ positive and $\mathrm{B}$ negative renal transplant recipients. Transplantation 1991, 51:391-396.

38. Fornairon S, Pol S, Legendre C, et al.: The long-term virological and pathologic impact of renal transplantation on chronic hepatitis B virus infection. Transplantation 1996, 62:297-299.

39. Mathurin P, Mouquet C, Poynard T, et al.: Impact of hepatitis B and $\mathrm{C}$ virus on kidney transplantation outcome. Hepatology 1999, 29:257-263.

40. Chan TM, Fang GX, Tang CS, et al.: Preemptive lamuvidine therapy based on HBV DNA level in HBsAg-positive kidney allograft recipients. Hepatology 2002, 36:1246-1252.

41. Terrault NA, Adey DB: The kidney transplant recipient with hepatitis $\mathrm{C}$ infection: pre- and post-transplantation treatment. Clin J Am Soc Nephrol 2007, 2:563-575.

42. Han DJ, Kim TH, Park SK, et al.: Results on preemptive or prophylactic treatment of lamivudine in $\mathrm{HBsAg}+$ renal allograft recipients: comparison with salvage treatment after hepatic dysfunction with HBV recurrence. Transplantation 2001, 71:387-394.

43. Ahn HJ, Kim MS, Kim YS, et al.: Clinical outcome of renal transplantation in patients with positive pretransplant hepatitis B surface antigen. J Med Virol 2007, 79:1655-1663.

44. - Degertekin B, Lok AS: Indications for therapy in hepatitis B. Hepatology 2009, 49:S129-S137. This article is an informative review that discusses the various clinical and virological indications for treatment in different patient populations.

45. Lok AS, McMahon BJ. Chronic hepatitis B [published erratum appears in Hepatology 2007, 45:1347]. Hepatology 2007, 45:507-539.

46. Chen CJ, Yang HI, Su J, et al.: Risk of hepatocellular carcinoma across a biological gradient of serum hepatitis B virus DNA level. JAMA 2006, 295:65-73.

47. Huang YW, Liu CJ, Lai MY, et al.: Discontinuation of lamivudine treatment for hepatitis flare after kidney or heart transplantation in hepatitis B surface antigen-positive patients: a retrospective case series. Clin Ther 2006, 28:1327-1334.

48. Chan TM, Tse KC, Tang CS, et al.: Prospective study on lamivudine-resistant hepatitis B in renal allograft recipients. Am J Transplant 2004, 4:1103-1109.

49. Tse KC, Yap DY, Tang CS, et al.: Response to adefovir or entecavir in renal allograft recipients with hepatitic flare due to lamivudine-resistant hepatitis B. Clin Transplant 2009 (Epub ahead of print).

50. Tse KC, Yung S, Tang C, et al.: Management of hepatitis B reactivation in patients with lupus nephritis. Rheumatol Int 2009, 29:1273-1277. 